\title{
The Use of Gabapentin in the Treatment of Neurogenic Cough: A Case Report and Review
}

\author{
Veronica del Calvo ${ }^{1}$, Alexandra Lackey ${ }^{1}$ and John $\mathrm{Li}^{2 *}$ \\ ${ }^{1}$ Medical Student, Florida State University College of Medicine, USA \\ ${ }^{2}$ Otolaryngology, Ear, Nose and Throat Associates of South Florida, USA
}

Submission: March 09, 2018; Published: March 14, 2018

*Corresponding author: John Li, Ear, Nose and Throat Associates of South Florida, P.A, USA, Tel: +(561) 748-4445; Email: Li@Dr-Li.net

\begin{abstract}
Cough is one of the most common chief complaints presenting for medical evaluation. While the diagnoses of upper respiratory infection (URI), postnasal drip, asthma, and gastroesophageal reflux (GERD) are easily made and easily treated, there are many cases that remain difficult to diagnose and treat. This study highlights a difficult case of intractable cough, and its resolution. The objective of this study is to draw attention to the diagnosis of Sensory Neuropathic Cough and update the diagnostician with an expanded armamentarium of tools for treatment of the difficult cough patient. Sensory neuropathic cough (SNC) is a commonly overlooked diagnosis that can be successfully treated with Gabapentin class medications.
\end{abstract}

Keywords: Neurogenic Cough; Gabapentin; Sensory Neuropathic Cough; Chronic Cough; Laryngeal Sensory Neuropathy, Vagal Neuropathy

Abbreviations: SNC: Sensory neuropathic cough; URI: Upper Respiratory Infection; GERD: Gastroesophageal Reflux; TCA: Tricyclic antidepressants

\section{Introduction}

Acute cough is usually a self-limited entity that resolves spontaneously within three weeks. It is usually associated with URI and is treated symptomatically with over-the-counter medications and rest. Chronic cough, defined as lasting greater than 8 weeks, is a much larger and more difficult problem and is the reason for many primary care visits all over the world. It is a symptom that is often associated with URI, postnasal drip, asthma, and GERD. These diagnoses are readily treated with successful resolution of the cough symptoms. However, there are many cases where the diagnosis is more difficult, and the treatments do not seem to work. Oftentimes, patients are seen by numerous providers without getting a definitive diagnosis. They would be tried on a variety of over-the-counter medications as well as the standard battery of prescription medications with little or no benefit.

One diagnosis of exclusion is Neurogenic Cough also known as Sensory Neuropathic Cough. One of the first to suggest the new diagnosis of SNC was Dr. Koufman J in 1999 who successfully treated a 19-year-old woman with chronic cough with gabapentin and amitriptyline [1].

SNC is believed to be the result of a decreased threshold in the vagal sensory afferent fibers. This increased nerve irritability results in a chronic, mysterious non productive cough that produces a non-diagnostic workup and fails to respond to conventional therapies. Unfortunately, for patients suffering from SNC, misdiagnoses are common. Unnecessary antibiotics, cough suppressants, inhalers, allergy medications, and proton pump inhibitors are prescribed which yield no relief. Some of these patients are submitted to a variety of testing including chest x-rays, upper endoscopy, and laryngoscopy, which yield no significant findings. Others are frustratingly diagnosed with psychogenic cough. This unique case corresponds to a 72-yearold Caucasian man with SNC who underwent extensive workup for non productive cough, but ultimately found relief with Gabapentin.

\section{Case Report}

A 72-year-old Caucasian male physician presented to our clinic with the chief complaint of a persistent non productive cough with progressive worsening. His cough began when he returned from a five week vacation to Europe 18 months prior to presentation. Over time, the cough became debilitating to his daily activities. He was waking up from sleep gasping for air. During the day, he would have numerous attacks where he could not catch his breath. He was previously evaluated by an internist, allergist, pulmonologist, gastroenterologist, and an outside otolaryngologist. Attempted therapies consisted of antihistamines, proton pump inhibitors, nebulising treatments, multiple trials of antibiotics, and lifestyle modifications. The 


\section{Global Journal of Otolaryngology}

previous physician's all charted a grossly normal physical exam. Each specialist recognized that the presentation of the patient's cough was somewhat atypical, but continued to prescribe conventional therapies.

One month prior to presentation, the patient was hospitalized for three days after a severe coughing episode. He received an extensive workup which included a CT scan of his sinuses, viral panels; legionnaire's disease testing, pulmonary function test, and a barium swallow study. All results were negative. Although he never received a definitive diagnosis of COPD, the patient was treated as if he had COPD exacerbation. The treatment did not provide him with answers or relief. Upon presentation to our clinic, the patient was extremely frustrated with his lack of progress and lack of symptom control. As a physician himself, he was surprised that the diagnosis and management of such a common symptom as cough could be so elusive. The review of systems was notable only for the chronic cough. He was not taking any relevant medications. There was no history of smoking or significant alcohol usage. There were no medical conditions that could be attributed to his cough except for a history a cardiac ablation for a trial fibrillation in 2016.

The family history is notable for the patient's father having similar coughing spells at night, but notes the father was a smoker and was overweight. The physical examination showed a normal otolaryngologic exam. The nasal passages were essentially clear, without pus or mucous. The larynx appeared to be normal, with normal vocal cord mobility and no significant reflux changes. There were no obvious masses or polyps. In view of the relatively normal exam, the duration of the symptoms and the failure to respond to standard treatment for asthma, COPD, GERD, sinusitis, etc., the common diagnoses were ruled out. A provisional diagnosis of Sensory Neuropathic Cough was made. A trial of Gabapentin was suggested. The patient was started on 100 mg of Gabapentin 3x/day for one week. During this week, the intensity and frequency of the cough significantly decreased.

The treatment regimen was subsequently reduced to 100 mg of Gabapentin 2x/day for one week, followed by $100 \mathrm{mg} \mathrm{1x/}$ day in the evening for two weeks. The regimen was eventually discontinued after initial resolution of his cough. The patient was symptom free for four weeks when the symptoms returned with the same intensity as before. The patient resumed Gabapentin $100 \mathrm{mg}$ in the evening for another two weeks. This was successful in resolving his symptoms. At this point, he discontinued the medications and has not had a recurrence.

\section{Discussion \& Literature Review}

Bastian, Vaidya, \& Delsupehe, in 2006, noted patients presenting with SNC can vary widely in severity. The cough can occur from a few times a day to hundreds of debilitating episodes per day. Associated symptoms may include hoarseness, stridor, and wheezing [2]. The cough may have a barking characteristic. In severe cases, the individual may have violent attacks leading to complications such as broken ribs, vomiting, or stress incontinence. This particular patient's attacks became so severe that he required hospitalization. Dr. Bastian suggested the unique characteristic of this type of cough is that it is not provoked by a significant external stimulus, but rather by subcritical stimuli due to hypersensitivity of the vagal cough reflex afferents [3]. Under normal physiologic conditions, external stimuli may include post nasal drip, reactive airway, gastric acid, or toxic fumes and other inhaled irritants.

Altman KW, Noordzij JP, Rosen CA, Cohen S, Sulica L [4] Found that various studies seemed to support the observation that the pathology of SNC was similar to that of HIV peripheral neuropathy, and virus induced Guillain-Barre [4]. The common denominator was nerve damage. Many cases of SNC have been observed to present after a viral URI. This observed pattern allows experts to hypothesize a post-viral cause of neural damage similarly seen in post-herpetic neuralgia. In SNC, however, the outcome is not pain, but rather a cough due to nerve misfiring [4]. This irritant hypersensitivity occurs when normal neural physiologic parameters are obstructed. Triggers in SNC include seemingly normal activities such as talking, laughing, inhaling air, and singing [5]. Unfortunately, there is currently no specific test that measures the hypersensitivity of the sensory afferents, and hence, no specific test to make a diagnosis of SNC. Since patients with SNC have normal laryngeal motor function, videostroboscopy, electromyography, and flexible rhinolaryngoscopy typically reveal normal findings. There is limited research on the pathophysiology of SNC, so there is no gold standard diagnostic criterion in work up and treatment.

Various studies have been conducted in search of a treatment regimen that will provide relief to individuals suffering from SNC. Bastian ZJ et al. [3] performed a retrospective cohort case series consisting of 32 patients and studied the effectiveness of three medications known to treat neuropathic pain, amitriptyline, desipramine, and gabapentin. The study concluded all drugs had similar efficacy in improving the cough, but noted differences in dosing regimens that needed to be tailored to the individual [3]. Another randomized clinical trial performed by Jeyakumar, Brickman \& Haben [5], investigated the use of amitriptyline versus a cough suppressant. While none of the 28 subjects achieved complete resolution with codeine/guaifenesin, a majority of these patients resolved their symptoms with a 10 mg dose of amitriptyline [5]. Lastly, a randomized, double blind clinical trial performed by Ryan, Birring, \& Gibson [6], treated 62 patients with refractory chronic cough with Gabapentin $(n=32)$ and placebo $(n=30)$. It also concluded that neuropathic medications, in this study gabapentin, offered significant improvement in the patients' symptoms and quality of life [6].

As an anti-seizure drug, Gabapentin is used as adjunctive therapy for seizure disorders. It has been found to be useful as treatment for postherpetic neuralgia in adults. Off-label uses include alcohol dependence and withdrawal, pruritus, diabetic neuropathy, fibromyalgia syndrome, hiccups, hot flashes, neuropathic pain, restless leg syndrome and chronic 
refractory cough. Although Gabapentin has a similar chemical structure to the neurotransmitter GABA, it does not bind to the GABA receptors and does not affect the production or reuptake of GABA. The proposed mechanism of action involves binding to presynaptic voltage-gated calcium channels containing an alpha-2-delta-1 subunit that modulates the release of excitatory neurotransmitters which act by interacting with GABA-R or NMDA-R. This feature is what gives it the ability to attenuate pain and control epilepsy $[7,8]$. Other medications such as Tricyclic antidepressants (TCA's), amitriptyline and desipramine, can be also be used. However, they are on the American Geriatric Society's Beers Criteria list of potentially inappropriate medications for use in older adults.

These medications should probably be avoided in patients over the age of 65. The most serious side effects of TCA's include anticholinergic activity, and cardiotoxicity that may lead to fatal cardiac arrhythmias. TCA's have a narrower therapeutic index when compared to Gabapentin, making Gabapentin a safer and more convenient drug of choice. For these reasons, Gabapentin was chosen for our patient and should be considered the first line treatment for Sensory neuropathic cough.

\section{Conclusion}

Sensory neuropathic cough (SNC) can be a frustrating condition for patients and difficult to diagnose for clinicians. This diagnosis is often not made because many providers are not aware that this diagnosis exists. Furthermore, many physicians are not familiar or not comfortable with the use of Gabapentin, amitriptyline and desipramine. This case report demonstrates how the appropriate diagnosis of SNC can be made, and that Gabapentin can be useful in the treatment of SNC. The successful and safe use of Gabapentin in this case also helped confirm the diagnosis of SNC.

\section{References}

1. Koufman J (2018) Chronic Cough. The Voice Institute of New York, USA.

2. Bastian RW, Vaidya AM, Delsupehe KG (2006) Sensory neuropathic cough: a common and treatable cause of chronic cough. Otolaryngol Head Neck Surg 135(1): 17-21.

3. Bastian ZJ, Bastian RW (2015) The use of neuralgia medications to treat sensory neuropathic cough: our experience in a retrospective cohort of thirty-two patients. PeerJ 3: e816.

4. Altman KW, Noordzij JP, Rosen CA, Cohen S, Sulica L (2015) Neurogenic cough. Laryngoscope 125: 1675-1681.

5. Jeyakumar A, Brickman TM, Haben M (2006) Effectiveness of amitriptyline versus cough suppressants in the treatment of chronic cough resulting from postviral vagal neuropathy. Laryngoscope 116: 2108-2112.

6. Ryan NM, Birring SS, Gibson PG (2012) Gabapentin for refractory chronic cough: a randomised, double-blind, placebo-controlled trial. Lancet 380: 1583-1589.

7. Rose MA, Kam PC (2002) Gabapentin: pharmacology and its use in pain management. Anaesthesia 57: 451-462.

8. Sills GJ (2006) The mechanisms of action of Gabapentin and pregabalin. Curr Opin Pharmacol 6: 108-132.

\section{Your next submission with Juniper Publishers will reach you the below assets}

- Quality Editorial service

- Swift Peer Review

- Reprints availability

- E-prints Service

- Manuscript Podcast for convenient understanding

- Global attainment for your research

- Manuscript accessibility in different formats ( Pdf, E-pub, Full Text, Audio)

- Unceasing customer service

Track the below URL for one-step submission https://juniperpublishers.com/online-submission.php 